\title{
Importance of Spin-Orbit Interaction for the Electron Spin Relaxation in Organic Semiconductors
}

\author{
L. Nuccio, ${ }^{1,2, *}$ M. Willis, ${ }^{1}$ L. Schulz, ${ }^{2}$ S. Fratini, ${ }^{3}$ F. Messina, ${ }^{4}$ M. D’ Amico, ${ }^{4,5}$ F. L. Pratt, ${ }^{6}$ J. S. Lord, ${ }^{6}$ I. McKenzie, ${ }^{6}$ \\ M. Loth, ${ }^{7}$ B. Purushothaman, ${ }^{7}$ J. Anthony, ${ }^{7}$ M. Heeney, ${ }^{8}$ R. M. Wilson, ${ }^{9}$ I. Hernández, ${ }^{1,10}$ M. Cannas, ${ }^{4,5}$ K. Sedlak, ${ }^{11}$ \\ T. Kreouzis, ${ }^{1}$ W. P. Gillin, ${ }^{1}$ C. Bernhard, ${ }^{2}$ and A. J. Drew ${ }^{1,6, \dagger}$ \\ ${ }^{1}$ Queen Mary University of London, School of Physics and Astronomy, Mile End Road, London E1 4NS, United Kingdom \\ ${ }^{2}$ Department of Physics and Fribourg Center for Nanomaterials, University of Fribourg, Chemin du Musée 3, \\ CH-1700 Fribourg, Switzerland \\ ${ }^{3}$ Institut Néel-CNRS and Université Joseph Fourier, Boîte Postale 166, F-38042 Grenoble Cedex 9, France \\ ${ }^{4}$ University of Palermo, Department of Physics and Chemistry, Via Archirafi 36, 90123 Palermo, Italy \\ ${ }^{5}$ Consorzio Nazionale Interuniversitario per le Scienze Fisiche della Materia-MeSIAM, Via Archirafi 36, 90123 Palermo, Italy \\ ${ }^{6}$ ISIS Pulsed Neutron and Muon Source, Rutherford Appleton Laboratory, Chilton, Didcot OX11 OQX, United Kingdom \\ ${ }^{7}$ Department of Chemistry, University of Kentucky, Lexington, Kentucky 40506-0055, USA \\ ${ }^{8}$ Department of Chemistry and Centre for Plastic Electronics, Imperial College London, SW7 2AZ London, United Kingdom \\ ${ }^{9}$ Queen Mary University of London, School of Engineering and Materials Sciences, Mile End Road, London E1 4NS, United Kingdom \\ ${ }^{10}$ MALTA Consolider Team, Departamento CITIMAC, Universidad de Cantabria, Avenida Los Castros s/ $n$, 39005 Santander, Spain \\ ${ }^{11}$ Laboratory for Muon-Spin Spectroscopy, Paul Scherrer Institute, CH-5232 Villigen PSI, Switzerland
}

(Received 29 November 2012; published 23 May 2013)

\begin{abstract}
Despite the great interest organic spintronics has recently attracted, there is only a partial understanding of the fundamental physics behind electron spin relaxation in organic semiconductors. Mechanisms based on hyperfine interaction have been demonstrated, but the role of the spin-orbit interaction remains elusive. Here, we report muon spin spectroscopy and time-resolved photoluminescence measurements on two series of molecular semiconductors in which the strength of the spin-orbit interaction has been systematically modified with a targeted chemical substitution of different atoms at a particular molecular site. We find that the spin-orbit interaction is a significant source of electron spin relaxation in these materials.
\end{abstract}

In recent years, there has been a considerable effort to understand spin dynamics of charge carriers in organic semiconductors (OSCs). Although current microelectronics is mainly founded on inorganic semiconductors, OSCs have several advantages, including the possibility to chemically tune their structure and electronic properties, the ability of self-assembly and mechanical flexibility. These properties open the way to large-area and low cost electronic applications [1-4]. But the property that most of all has boosted their use in the field of spin-based electronics (spintronics) is their very long spin relaxation time, which can exceed the typical times observed in inorganic materials by orders of magnitude $[5,6]$. Organic spintronic devices operation relies in fact on the efficient propagation of spin-polarized carriers through the OSC [7]. The archetypical example of an organic spintronic device is the spin valve [8], where an OSC layer separates two ferromagnetic electrodes. Spin relaxation in the OSC causes a progressive loss of spin polarization, and therefore it has a detrimental effect on device performance, limiting the magnitude of the magnetoresistance that can be achieved in these devices. It is therefore very important to understand the mechanisms of spin relaxation in OSCs.

Starting from the observation that OSCs are mainly composed of light elements and as a consequence the spin-orbit interaction (SOI) is small, great attention has been paid to the hyperfine interaction (HFI). Theories for spin relaxation in OSCs based on the HFI have been developed, while the SOI has been less considered [9-12]. According to Ref. [11], the spin of mobile charge carriers precesses around the local field given by the sum of the external field and the random hyperfine fields from the surrounding nuclear spins. The hopping of the charge carriers to different sites, together with this precession that is different for every site, gives rise to spin relaxation. Deuteration experiments on spin valves and organic light emitting diodes (OLEDs), whose behavior is also dependent on spin dynamics despite the fact that they do not include any magnetic material [13], support this assumption [14-16].

In addition to the HFI-based spin relaxation mechanism there may be an additional significant contribution from the SOI. The occurrence of sizeable SOI-based spin relaxation in light organic materials has already been demonstrated in, for example, carbon nanotubes or graphene $[17,18]$. A spin relaxation in OSCs based on SOI has been considered by some authors [19-21]. On the experimental side, it has been shown that the magnetic field dependent luminescence and current efficiencies in OLEDs are significantly altered by heavier species [22,23], supporting the 
relevance of SOI. However, direct evidence of a SOI-based spin relaxation is still limited. Electron paramagnetic resonance (EPR) measurements on tris(8-hydroxyquinolinato) aluminium $\left(\mathrm{Al}_{3}\right)$ show a signal consistent with a quasifree electron. Although the unstructured nature of the signal does not allow us to assess the magnitude of the two interactions, the observed anisotropy in the $g$ factor is compatible with the presence of a weak SO interaction [24]. Furthermore, it has been pointed out that the $g$ factor deviation comes from spin mixing between different orbital states, but does not include contributions from the spin mixing within the same orbital. As a consequence, the $g$ factor may not be a good measure of the spin mixing and thus severely underestimate the SOI in these materials [21]. A more detailed discussion of the existing EPR data is reported in the Supplemental Material [25].

In the present work we provide direct evidence, using combined information from muon spin resonance ( $\mu \mathrm{SR})$ and time-resolved photoluminescence spectroscopy, of the existence of a sizable SOI-based mechanism for the electron spin relaxation in OSCs.

Our strategy to differentiate between the SOI and HFI contributions to the spin relaxation is to perform targeted chemical substitutions within the OSC, by replacing an atom with a heavier one. This leads to a systematic modification of the SOI, which depends on the atomic number $Z$. The corresponding changes in the spin and nuclear magnetic moments are not correlated with $Z$.

Spin-polarized muons have been shown to be a sensitive probe for the electron spin relaxation rate (eSR) in OSCs $[26,27]$. In particular, we report measurements of avoided level crossing (ALC) resonances, whose amplitude is essentially proportional to the eSR.

In this type of experiment $100 \%$ spin-polarized muons are implanted into the OSC. During the thermalization process, a proportion of the muons capture an electron from a molecule forming a hydrogenlike species, called muonium. Muonium can then bind to a molecule. In an ALC experiment a magnetic field is applied parallel to the initial muons spin direction. At a particular value of the magnetic field, due to cross relaxation effects, a depolarization of the muon spin occurs, and gives rise to the observed ALC resonances, even in the absence of eSR $[28,29]$. Since the spins of the electron and of the muon in muonium are coupled, if an electron spin relaxation mechanism is effective in the material it causes a further relaxation of the muon spin. This is detected as an increase in amplitude of the ALC resonance [26,27] (more detailed information about the technique is reported in Ref. [26] and the Supplemental Material [25]). A quantitative estimation of the eSR at room temperature can be obtained by modelling of the ALCs as described in the literature $[26,27,30] . \mu \mathrm{SR}$ is a spectroscopic technique that measures the eSR directly and in the bulk of the OSCs, thus avoiding the complications of measurements on OLEDs or spin valves where additional influences, for example, from the interfaces, need to be considered. As a consequence $\mu \mathrm{SR}$ is a very good probe of the physics intrinsic to OSCs.

Our $\mu \mathrm{SR}$ experiments were performed at the ALC instrument at the Paul Scherrer Institute, and at the HIFI instrument at ISIS. The ALCs were measured in $\mathrm{Al}_{3}$, a material widely used both in spintronics and OLEDs applications [31-34] and in $\mathrm{Ga} q_{3}$, In $q_{3}$, and $\mathrm{Biq}_{3}$, three samples of analogous molecules in which the aluminium atom has been substituted (here named the $X q_{3}$ series). Their molecular structure is shown in Fig. 1(a). Al $q_{3}$ (99.995\% pure) was purchased from Sigma Aldrich. $\mathrm{Ga} q_{3}, \operatorname{In} q_{3}$, and $\mathrm{Bi} q_{3}$ were synthesized using a published method $[35,36]$. Al $q_{3}$, $\mathrm{Ga} q_{3}$ and $\operatorname{In} q_{3}$ were purified using train sublimation, resulting in a polycrystalline powder. $\mathrm{Bi} q_{3}$ was used as synthesized because it degrades if train sublimation is attempted.

We also measured the ALCs in a second series of molecules, consisting of triethylsilylethynyl anthradifuran (TES-ADF), triethylsilylethynyl anthradithiophene (TES-ADT), and triethylsilylethynyl anthradiselenophene (TES-ADS). These correspond to the central backbone containing oxygen, sulphur, or selenium, respectively, on both of its end rings [Fig. 1(b)]. TES-ADF, TES-ADT, and TES-ADS (here named the TES series) were synthesized and purified according to a published procedure $[37,38]$.

Figure 2 shows the ALCs as measured in the $X q_{3}$ series at 10 (blue triangles) and $300 \mathrm{~K}$ (red circles). The black lines in Fig. 2 are the results of the modeling through which the eSR values are determined [26,27,30]. It is immediately clear that there is a strong dependence of the ALC amplitude on temperature, with the ALCs at $10 \mathrm{~K}$ having a significantly smaller amplitude compared with those at $300 \mathrm{~K}$. This indicates that the eSR increases at high temperature. This is consistent with our previous work, which showed that in several organic compounds, including $\mathrm{Ga} q_{3}$, the eSR is thermally activated with an energy scale comparable to molecular vibrations [26]. Moreover, it also appears from Fig. 2 that the magnitude of the ALCs at $300 \mathrm{~K}$ increases systematically with $Z$, whereas at $10 \mathrm{~K}$ it is significantly smaller and independent of $Z$. Based on the previously discussed relationship between the magnitude
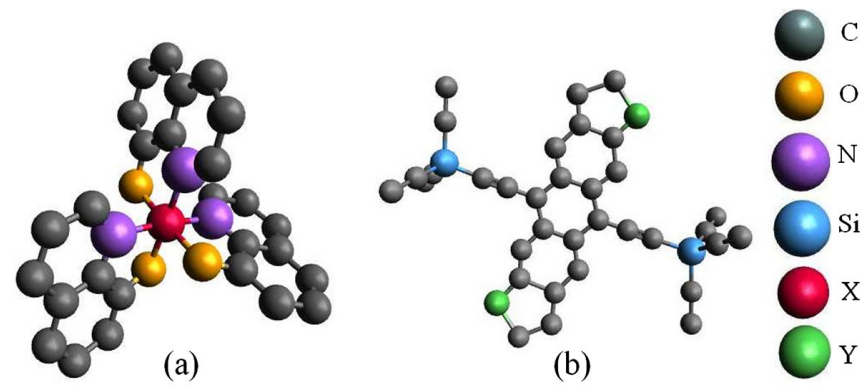

FIG. 1 (color online). Molecular structures of (a) the $X q_{3}$ series, where $X=\mathrm{Al}, \mathrm{Ga}, \mathrm{In}, \mathrm{Bi}$, and (b) the TES series, where $Y=\mathrm{O}, \mathrm{S}$, Se. Hydrogen atoms are not shown for clarity. 


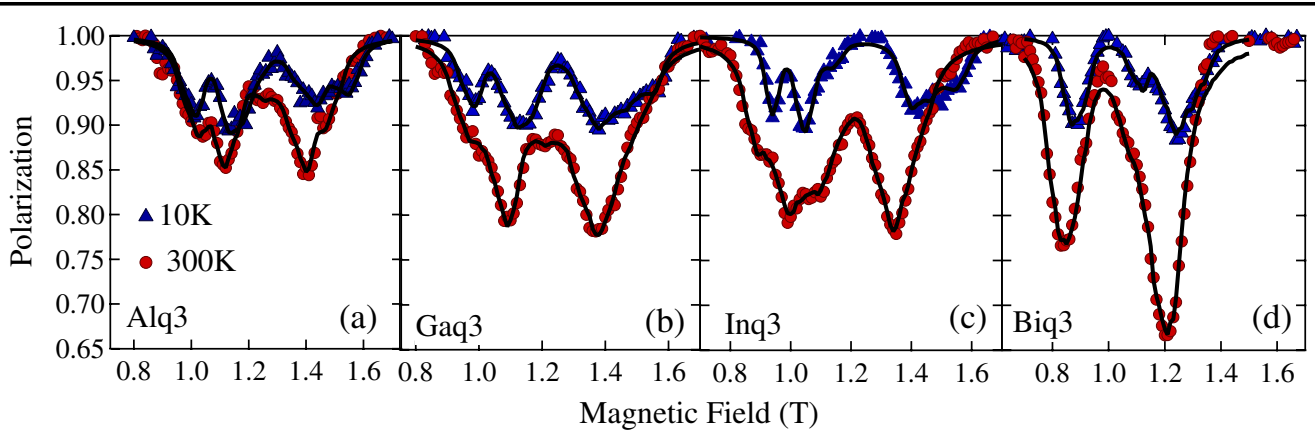

FIG. 2 (color online). Muon spin polarization around the ALCs in (a) $\mathrm{Al} q_{3}$, (b) Ga $q_{3}$, (c) In $q_{3}$, and (d) Bi $q_{3}$ at $10 \mathrm{~K}$ (blue triangles) and $300 \mathrm{~K}$ (red circles). Modelling for these ALCs is indicated by the black lines and is used to determine the electron spin relaxation rate, which is essentially proportional to the amplitude of the ALC curves (see text).

of the ALCs and the eSR, this points to a thermally activated, SOI-based spin relaxation mechanism.

Such an increase of the eSR with the $Z$ of the substituent atom, the so-called heavy atom effect, is expected for a SOI-based spin relaxation mechanism. However, we do not expect a simple relationship between these two quantities, for the following reasons. First of all, although the atomic SO coupling constant is predicted to increase as $Z^{2}$ based on simplifying assumptions [39], its actual value varies in a complex way with $Z$ [40]. Second, the effect of the SOI due to the substituted atom compared to that from all the other atoms in the molecule depends on the weight of the wave function at the substituent site, and this is molecule specific [20]. To overcome these difficulties, and estimate independently the strength of the SOI along the series in order to relate it with the eSR rate, we have measured the exciton singlet to triplet conversion rate, known as the intersystem crossing rate $\left(k_{\mathrm{ISC}}\right)$, via time-resolved photoluminescence. An estimation of $k_{\mathrm{ISC}}$ has been possible in $\mathrm{Al} q_{3}, \mathrm{Ga} q_{3}$, and $\operatorname{In} q_{3}$ where singlet and triplet emissions can be both clearly singled out by time-resolved luminescence measurements (details of these measurements are shown in the Supplemental Material [25]). This was not possible in $\mathrm{Bi} q_{3}$ and in the TES series due to their different optical properties.

The $k_{\text {ISC }}$ is proportional to the square of the matrix element of the SOI Hamiltonian between the singlet and triplet states [41], so $k_{\mathrm{ISC}}$ can be used as a reliable measurement of the strength of the molecular SOI. This approach is solidly founded on the widespread understanding of the physics behind the singlet-to-triplet conversion [41-44], and allows us to bypass the fact that the precise form of the underlying dependence of the eSR on the atomic number of the substituents is unknown.

Figure 3(a) shows the eSR as measured through $\mu \mathrm{SR}$ as a function of $k_{\mathrm{ISC}}$, which is proportional to the SOI strength, in the $X q_{3}$ series. It reveals a clear relationship between the eSR and the strength of the SOI. This dependence suggests the existence of a SOI-driven mechanism for spin relaxation. We also plot, in Fig. 3(b) the same eSR data versus the $Z$ of the substituent atom. The expected increase of the eSR with $Z$ is indeed observed in Fig. 3(b).
In the following, we will show that an alternative interpretation in terms of a HFI-based mechanism does not provide a consistent explanation of the observed change of eSR with $Z$.

Given that the only change we made to the molecule was the central atom, with the number and location of hydrogen atoms remaining unaltered, there should not be any direct effect of the HFI between the electron and hydrogen in the changes observed here.

An effect of the HFI due to changes to the spin and nuclear magnetic moment of the central atom is also very unlikely. Table I shows that the magnitude of the spin and the nuclear moment of the substituent atoms does not correlate with the observed increase of the eSR. Instead, for the case of $\mathrm{Al}_{3}$ and $\mathrm{Ga} q_{3}$, where the eSR roughly doubles, both the nuclear moment and spin of $\mathrm{Al}$ are significantly larger than those of Ga. Finally, one might argue that changing the central atoms may still bear an indirect effect on the HFI that could influence the observed results. Changing the mass of an atom in the molecule can imply a change in the bond lengths and angles or, more generally, a modification of the energy and population of vibrational modes. Both of these effects could result in a
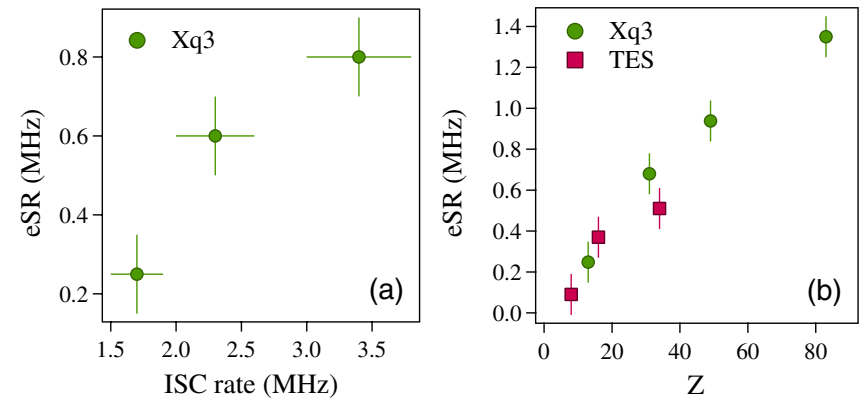

FIG. 3 (color online). (a) Electron spin relaxation rate as a function of the intersystem crossing rate in the $X q_{3}$ series at $300 \mathrm{~K}$. The electron spin relaxation rate shows a dependence on the intersystem crossing rate, which is used here as a measurement of the strength of the spin-orbit interaction. (b) eSR for the $X q_{3}$ and TES series as a function of the atomic number of the substituent atom, $Z$. 
TABLE I. Isotope, natural abundance, nuclear spin, and magnetic moment for the substituent elements in $X q_{3}$ and TES, and eSR in the corresponding molecules.

\begin{tabular}{llccc}
\hline \hline & \multicolumn{1}{c}{ Isotope (\%) } & Spin & $\begin{array}{c}\text { Nuclear moment } \\
\left(\mu / \mu_{N}\right)\end{array}$ & $\begin{array}{c}\text { eSR in related } \\
\text { molecule (MHz) }\end{array}$ \\
\hline $\mathrm{Al}$ & ${ }^{27} \mathrm{Al}(100 \%)$ & $5 / 2$ & 3.64 & 0.25 \\
$\mathrm{Ga}$ & ${ }^{69} \mathrm{Ga},{ }^{71} \mathrm{Ga}(60 \%, 40 \%)$ & $3 / 2,3 / 2$ & $2.02,2.56$ & 0.68 \\
$\mathrm{In}$ & ${ }^{113} \mathrm{In},{ }^{115} \mathrm{In}(4.3 \%, 95.7 \%)$ & $9 / 2,9 / 2$ & $5.52,5.54$ & 0.94 \\
$\mathrm{Bi}$ & ${ }^{209} \mathrm{Bi}(100 \%)$ & $9 / 2$ & 4.11 & 1.35 \\
$\mathrm{O}$ & ${ }^{16} \mathrm{O},{ }^{17} \mathrm{O},{ }^{18} \mathrm{O}(99.76 \%, 0.04 \%, 0.2 \%)$ & $0,5 / 2,0$ & $0,1.89,0$ & 0.09 \\
$\mathrm{~S}$ & ${ }^{32} \mathrm{~S},{ }^{33} \mathrm{~S},{ }^{34} \mathrm{~S},{ }^{36} \mathrm{~S}(94.9 \%, 0.8 \%, 4.3 \%, 0.02 \%)$ & $0,3 / 2,0,0$ & $0,0.64,0,0$ & 0.37 \\
$\mathrm{Se}$ & ${ }^{74} \mathrm{Se},{ }^{76} \mathrm{Se},{ }^{78} \mathrm{Se},{ }^{80} \mathrm{Se},{ }^{82} \mathrm{Se},{ }^{77} \mathrm{Se}$ & $0,0,0,0,0,1 / 2$ & $0,0,0,0,0,0.53$ & 0.51 \\
& $(0.9 \%, 9.4 \%, 23.8 \%, 49.6 \%, 8.7 \%, 7.6 \%)$ & & & \\
\hline \hline
\end{tabular}

modification of the electron-hydrogen HFI. However, it is important to note that any changes would be specific to the particular geometry of the molecule. We therefore measured the eSR in a second series of molecules with a different structure, the above mentioned TES series. The raw muon spectra for the TES series are shown in the Supplemental Material [25], where the same qualitative behavior as for the $X q_{3}$ series can be observed.

The values of the eSR rate obtained for the TES series at $300 \mathrm{~K}$ are given in Fig. 3(b) (squares) and Table I, together with the values from the $X q_{3}$ series for comparison. As already observed for the $X q_{3}$ series, also for the TES we measure an increase in the eSR along the series. Remarkably, the behavior of the eSR for the TES series is very similar to the one observed in the $X q_{3}$ series. Given that any changes to the hydrogen $\mathrm{HF}$ fields experienced by the electron deriving from changes in bond lengths or vibrational modes would be specific to each series, the comparison presented in Fig. 3(b) indicates that it is likely that any indirect effect of changing the mass on the hydrogenelectron HFI is not the cause of the observed changes in the eSR. This is confirmed by the fact that any effect due to the change of bond angles or length should be also visible in the $10 \mathrm{~K}$ data, which are instead comparable in all the samples within both series. We also note that the majority isotopes of all materials substituted in the TES series have zero nuclear spin (see Table I), which excludes any direct HFI effect of the heavy atom in this second set of molecules.

The correlation between SOI strength and eSR, its dependence on the $Z$ of the substituted atom and the similarities between the two series lead us to conclude that the observed change in the eSR upon substitution of an atom is indeed governed by the SOI.

As previously mentioned, the $Z$-dependent spin relaxation mechanism observed here is temperature dependent. In particular, if one looks at Fig. 2 it becomes clear that the amplitude of the ALCs, and as a consequence the eSR, shows a dependence on $Z$ only at $300 \mathrm{~K}$. At $10 \mathrm{~K}$, the ALCs of all the samples have comparable amplitudes. This implies in turn that if a spin relaxation mechanism is effective at low temperatures, it is not SOI driven.
The data on the TES series allow us to stress another important point. Based on the data in Fig. 3(a) one could raise the question of whether the effects of the SOI are relevant only for molecules with heavy atoms, such as $\mathrm{Ga}$ and In. If one looks at the eSR values in TES [Fig. 3(b) and Table I], it can be immediately seen that there is a significant change in eSR moving from oxygen to sulphur, with the atomic number of sulphur being very close to that of aluminium. It can hence be concluded that this SOI-based mechanism is not only limited to molecules including elements with high atomic numbers, but it is already effective in the presence of atoms with atomic numbers in the range of $\mathrm{Al}$ or $\mathrm{S}$, which are commonly found in small molecule semiconductors and polymers used in organic electronics and spintronics. On the other hand, based on the present results, we cannot estimate the relevance of this SOI-based electron spin relaxation mechanism in molecules with no elements heavier than oxygen.

Finally, we note that the values of the eSR at $300 \mathrm{~K}$ $(\sim 1 \mathrm{MHz})$ are in agreement with the commonly quoted spin relaxation times in these molecules (of the order of $1 \mu \mathrm{s}$ [6]). As a consequence the contribution of the SOI to the electron spin relaxation in these materials is significant and cannot be neglected. Further details of the mechanism remain to be elucidated by future theoretical and experimental studies.

In summary, we have directly measured through $\mu \mathrm{SR}$ the electron spin relaxation rate in $\mathrm{Al}_{3}$ and analogous molecules containing heavier species, and we have shown that it depends on the strength of the spin-orbit interaction, probed through the intersystem crossing rate.

We have hence given direct evidence that, besides the HFI-based mechanisms for spin relaxation that have previously been demonstrated in OSCs, a SOI-based mechanism of relevant magnitude also needs to be considered.

A.J.D. acknowledges financial support from the Leverhulme Trust and the EPSRC (Grant No. EP/ G054568/1). M.D'A. acknowledges financial support from CNISM, project "Analisi spettroscopica di materiali di interesse applicativo in optoelettronica, nelle telecomunicazioni e in dosimetria retrospettiva" and Professor F. Gelardi for useful discussions. L. N., F. M., M. D'A., 
and M. C. acknowledge partial financial support from project “P. O. R. Regione Sicilia-Misura 3.15-Sottoazione C." L. S. and C. B. acknowledge financial support from the SNF Grant No. 20020-140225, and the NCCR program MaNEP. I. H. acknowledges RAEng/EPSRC and FP7 (MC-CIG).

*laura.nuccio@unifr.ch

†A.J.Drew@qmul.ac.uk

[1] D. Voss, Nature (London) 407, 442 (2000).

[2] C. Reese, M. Roberts, M.-m. Ling, and Z. Bao, Mater. Today 7, 20 (2004).

[3] S. R. Forrest, Nature (London) 428, 911 (2004).

[4] R. W. I. de Boer, M.E. Gershenson, A.F. Morpurgo, and V. Podzorov, Phys. Status Solidi (a) 201, 1302 (2004).

[5] S. Sanvito, Nat. Mater. 6, 803 (2007).

[6] V. A. Dediu, L. E. Hueso, I. Bergenti, and C. Taliani, Nat. Mater. 8, 707 (2009).

[7] A. J. Drew et al., Nat. Mater. 8, 109 (2008).

[8] Z.H. Xiong, D. Wu, Z. V. Vardeny, and J. Shi, Nature (London) 427, 821 (2004).

[9] P. A. Bobbert, T. D. Nguyen, W. Wagemans, F. W. A. van Oost, B. Koopmans, and M. Wohlgenannt, Synth. Met. 160, 223 (2010).

[10] P. A. Bobbert, Nat. Mater. 9, 288 (2010).

[11] P. A. Bobbert, W. Wagemans, F. W. A. vanOost, B. Koopmans, and M. Wohlgenannt, Phys. Rev. Lett. 102, 156604 (2009).

[12] J. J. H. M. Schoonus, P. Lumens, W. Wagemans, J. Kohlhepp, P. Bobbert, H. Swagten, and B. Koopmans, Phys. Rev. Lett. 103, 146601 (2009).

[13] J. Kalinowski, M. Cocchi, D. Virgili, P. Di Marco, and V. Fattori, Chem. Phys. Lett. 380, 710 (2003).

[14] T. D. Nguyen, G. Hukic-Markosian, F. Wang, L. Wojcik, X.-G. Li, E. Ehrenfreund, and Z. V. Vardeny, Nat. Mater. 9, 345 (2010).

[15] T. D. Nguyen, G. Hukic-Markosian, F. Wang, L. Wojcik, X.-G. Li, E. Ehrenfreund, and Z. V. Vardeny, Synth. Met. 161, 598 (2011).

[16] T.D. Nguyen, T.P. Basel, Y.-J. Pu, X-G. Li, E. Ehrenfreund, and Z. V. Vardeny, Phys. Rev. B 85, 245437 (2012).

[17] T. Ando, J. Phys. Soc. Jpn. 69, 1757 (2000).

[18] Wei Han, K. M. McCreary, K. Pi, W. H. Wang, Y. Li, H. Wen, J. R. Chen, and R. K. Kawakami, J. Magn. Magn. Mater. 324, 369 (2012).

[19] S. Bandyopadhyay, Phys. Rev. B 81, 153202 (2010).

[20] Z. G. Yu, Phys. Rev. Lett. 106, 106602 (2011).

[21] Z. G. Yu, Phys. Rev. B 85, 115201 (2012).
[22] P.E. Burrows, L.S. Sapochak, D. M. McCarty, S.R. Forrest, and M.E. Thompson, Appl. Phys. Lett. 64, 2718 (1994).

[23] P. Shakja, P. Desai, M. Somerton, G. Gannaway, T. Kreouzis, and W.P. Gillin, J. Appl. Phys. 103, 103715 (2008).

[24] M. N. Grecu, A. Mirea, C. Ghica, M. Cölle, and M. Schwoerer, J. Phys. Condens. Matter 17, 6271 (2005).

[25] See Supplemental Material at http://link.aps.org/ supplemental/10.1103/PhysRevLett.110.216602 for details of the experimental techniques and additional data.

[26] L. Schulz et al., Phys. Rev. B 84, 085209 (2011).

[27] L. Nuccio, L. Schulz, M. Willis, F. L. Pratt, M. Heeney, N. Stingelin, C. Bernhard, and A. J. Drew, J. Phys. Conf. Ser. 292, 012004 (2011).

[28] B. D. Patterson, Rev. Mod. Phys. 60, 69 (1988).

[29] S. R. Kreitzman and E. Roduner, Chem. Phys. 192, 189 (1995).

[30] J. S. Lord, Physica (Amsterdam) 374B, 472 (2006).

[31] L. Schulz et al., Nat. Mater. 10, 39 (2010).

[32] Z. L. Shen, P.E. Burrows, V. Bulović, S. R. Forrest, and M. E. Thompson, Science 276, 2009 (1997).

[33] C. W. Tangand and S. A. Vanslyke, Appl. Phys. Lett. 51, 913 (1987).

[34] Z.H. Xiong, D. Wu, Z. V. Vardeny, and J. Shi, Nature (London) 427, 821 (2004).

[35] H. Schmidbaur, J. Lettenbauer, D. L. Wilkinson, G. Muller, and O. Kumberger, Naturforsch. 46, 901 (1991).

[36] J. N. Petronio and W. E. Ohnesorge, Anal. Chem. 39, 460 (1967).

[37] M. M. Payne, S. A. Odom, S. R. Parkin, and J. E. Anthony, Org. Lett. 6, 3325 (2004).

[38] J. E. Anthony and B. Purushothaman, in Organic FieldEffect Transistors, SPIE Proceedings (SPIE, Bellingham, WA, 2007), Vol. 6658, p. 6580L.

[39] L.D. Landau and E. M. Lifshits, Quantum Mechanics: Nonrelativistic Theory (Butterworth-Heinemann, London, 1977).

[40] M. Montalti, A. Credi, L. Prodi, and M. T. Gandolfi, Handbook of Photochemistry (Taylor \& Francis, London, 2006).

[41] D. Beljonne, Z. Shuai, G. Pourtois, and J. L. Bredas, J. Phys. Chem. A 105, 3899 (2001).

[42] E. A. Gastilovich, N. V. Korol'kova, V. G. Klimenko, and R. N. Nurmukhamentov, Opt. Spectrosc. 104, 491 (2008).

[43] V. G. Klimenko, E. A. Gastilovich, S. A. Serov, and R. N. Nurmukhametov, Opt. Spectrosc. 106, 483 (2009).

[44] M. Kasha, Discuss. Faraday Soc. 9, 14 (1950). 